\title{
LAS TITULACIONES DE EDUCACIÓN: UNA PERSPECTIVA HISTÓRICO-COMPARADA EN ESPAÑA Y EUROPA
}

(THE DEGREES IN EDUCATION: A HISTORICAL-COMPARATIVE PERSPECTIVE IN SPAIN AND EUROPE)

\author{
Joan María Senent \\ Universidad de Valencia
}

\section{RESUMEN}

El artículo plantea de entrada la historia de las titulaciones educativas en España desde sus inicios hace más de cien años hasta nuestra realidad universitaria en el inicio de una nueva reforma.

Posteriormente se entra en un análisis de las titulaciones educativas, excepto las concernientes con la formación del profesorado. En este estudio se analizan las titulaciones que tanto dentro de la universidad como desde los estudios superiores no universitarios se desarrollan en España, Francia, Italia, Bélgica, Alemania y Gran Bretaña.

Desde una óptica comparativa se obtienen conclusiones de este análisis, referidas especialmente a las estructuras universitarias, tipos de módulos, presencia de profesionales entre el profesorado, prácticas y característica de los diseños curriculares, así como se plantea posibles repercusiones.

\section{ABSTRACT}

The article deals with the history of the degrees in education in the Spanish university from its inception more than one hundred years ago, until our present university context in the beginning of a new reform.

The text analyses the university degrees, except those related to teacher training. The study tackles the degrees developed, both in the university and in other forms of tertiary education, in Spain, France, Italy, Belgium, Germany and the United Kingdom. 
Several conclusions are reached from a comparative approach. They are mainly refered to the structures of the universities, the different types of modules, the presence of professionals among teachers, the teaching practices, and the special features of curricular design. The article assesses the likely future impact of these elements in the degrees in education.

\section{DEFINIENDO EL TRABAJO}

En estos tiempos en que nos adentramos en una nueva reforma de los planes de estudio, parece el momento adecuado para hacer una reflexión sobre las titulaciones de educación en la universidad española y europea. Como nos ocurrió hace unos años cuando iniciamos los trabajos de los libros blancos impulsados por la ANECA, abordar en general las titulaciones de educación en la enseñanza superior europea es abrir un panorama de más de un centenar de titulaciones con perfiles bastante diversos que desembocan en áreas de trabajo diferentes.

Por eso parece conveniente realizar una primera opción en este análisis, -como a su vez se hizo hace algunos años con las Redes de Educación que surgieron de la convocatoria de la Aneca-, y establecer un objetivo más modesto, pero a su vez más realista, como es el abordar las titulaciones de educación que no tienen como objetivo directo la formación del profesorado, tanto el referido a la educación primaria como el de la educación secundaria.

En el contexto español, la opción anterior nos conduce a las titulaciones de Pedagogía, Psicopedagogía y Educación Social, dejando fuera del bloque los títulos de Magisterio en todas sus especialidades, así como la formación de los profesores de secundaria bien se a través del CAP, del TED o de los futuros Másteres de Formación del profesorado de educación secundaria.

\section{BREVE HISTORIA DE LAS TITULACIONES DE EDUCACIÓN EN LA UNIVERSIDAD ESPAÑOLA}

La historia de la titulación actual de Pedagogía se remonta a más de un centenar de años y ha sido descrita en diversas obras, especialmente por el prof. León Esteban (2007). Advertimos en su trabajo como los estudios de Pedagogía aparecen con la creación de la Sección Universitaria de Pedagogía en el seno de la Facultad de Filosofía y Letras, realizada por el Decreto de 27 de enero de 1932 (Gaceta 29/1/1932). Esta creación de la pedagogía como sección universitaria no fue repentina sino que culmina una serie de estudios pedagógicos que ya existían en la universidad española ${ }^{1}$ y que habían culminado con la creación de la Escuela Superior del Magisterio en 1909 (Real Decreto del 3 de junio de 1909).

La Sección de Pedagogía nace con cuatro objetivos bien planteados en su decreto de creación: 
a) Cultivo de las Ciencias de la Educación y desarrollo de los estudios de Pedagogía Superior.

b) Formación del profesorado de segunda enseñanza (hoy diríamos educación secundaria).

c) Formación de los Directores de Escuelas Graduadas.

Con la guerra civil de 1936, la mayoría del profesorado se exilia y en 1940, ya con el franquismo-, se reorganiza la Sección de Pedagogía de la Universidad de Madrid, aunque no tendrá un reconocimiento oficial hasta 1944. El título común es el de "Filosofía y Letras" que habilita para las oposiciones a cátedra y otros profesores de enseñanzas medias y Escuelas Normales, y es considerado un mérito, en su especialidad de Pedagogía para las cátedras de Escuelas normales y el cuerpo de dirección y de inspectores.

Posteriormente, la orden de 18/7/1955, establece el Plan de Estudios de la Facultad de Filosofía y Ciencias de la Educación con dos cursos comunes y tres de especialización, una de las cuales será la de Pedagogía. Este plan, con diferentes modificaciones parciales se mantendrán hasta 1973 y ofrecerá dentro de la sección de Pedagogía, tres modalidades: Organización y Administración Pedagógica, Educación Especial y Orientación Profesional y Escolar.

El Decreto 1974/73 de 12 de Julio (BOE 22/8/1973) autoriza la reestructuración de la Facultad de Filosofía y Letras, de la que van a surgir tres facultades: Geografía e Historia, Filología y Filosofía y Ciencias de la Educación en la que quedan los estudios pedagógicos.

Finalmente el Real Decreto 915/1992 de 17 de julio reorganiza los estudios estableciendo la Licenciatura en Pedagogía (primero más segundo ciclo, es decir 5 años) y la Licenciatura en Psicopedagogía (sólo $2^{\circ}$ ciclo, dos años) exigiendo haber cursado un primer ciclo en alguna de las anteriores carreras o en la de Magisterio. Asimismo, el R. D. 1420/1991 de 30 de Agosto, establece las directrices propias de la Diplomatura en Educación Social (primer ciclo, tres años) como una nueva titulación en el ámbito educativo. Más recientemente, en el año 2000 se modifican de nuevo los planes de estudios de estas titulaciones, realizándose cambios en el diseño curricular y especialmente en el creditaje de las materias, aunque no se modifica la estructura de las mismas.

Como se ha indicado la titulación de Educación Social nace hace solamente quince años como transformación en muchos casos de otras titulaciones en el ámbito de la formación profesional y de los estudios no formales, con el objetivo de preparar profesionales que intervengan en el ámbito de la educación no formal, especialmente en la educación especializada, la animación sociocultural y la educación de adultos, así como otros nuevos ámbitos que en estos años se han ido desarrollando. 


\section{LAS TITULACIONES DE LA EDUCACIÓN EN EL ÁMBITO EUROPEO}

Establecida una breve visión histórica de las titulaciones del ámbito educativo en el panorama español, el proceso iniciado en las universidades del continente siguiendo las pautas de Bolonia, nos fuerza a lanzar una mirada comparativa a la situación de esas mismas titulaciones educativas, cuya reforma se plantea actualmente en nuestro país. En la misma línea de la opción realizada al presentar la visión histórica, dejaremos de lado las titulaciones correspondientes a la formación del profesorado tanto en lo que respecta a la educación primaria como a la secundaria.

Una primera e interesante constatación a realizar es la diversidad de ubicación de esos estudios en Europa. Si en el caso español hemos hablado en todas las ocasiones de formaciones en la "universidad" como la cuna donde se han desarrollado los estudios pedagógicos e incluso los de formación del profesorado desde el inicio de los años 70, en muchos países europeos nos vamos a encontrar con una enseñanza superior no universitaria de bastante prestigio en la medida en que se trata de enseñanzas profesionalizadoras. En el ámbito pedagógico estas enseñanzas afectan a los estudios de formación del profesorado y los correspondientes a nuestra Educación Social, aún cuando con una gran heterogeneidad de denominaciones.

Voy a incluir en el análisis tanto estos últimos estudios como los realizados en la universidad, correspondientes a nuestra Pedagogía o Psicopedagogía, aún cuando también con un gran abanico de denominaciones.

Una segunda cuestión que deberemos sopesar es la situación de cambio, o de salir de un cambio, de muchas de las titulaciones presentadas. En efecto, la adaptación al Espacio Europeo de Educación Superior ha motivado en algunos países, haber realizado ya un cambio de sus estructuras siguiendo el esquema de Bolonia:

a) Grado - Bachelor - Licence - Laurea (180 (casi todos) ó 210/240 créditos (algunos)).

b) Maestría - Máster - Maitrîse (Profesional o de Investigación).

c) Doctorado.

Mientras en otros países europeos este cambio se está produciendo (o iniciando) en estos meses. Este cambio de estructuras ha supuesto en la mayor parte de los casos un proceso de modificación del diseño curricular de los planes de estudio, generalmente basado en un modelo de competencias, así como también en muchos países está implicando una profunda revisión metodológica que podría constituirse realmente en el verdadero cambio de la enseñanza superior europea (universitaria o no). 
Estas dos situaciones unidas a los propios cambios derivados del desarrollo tecnológico que sin duda afectan también a las titulaciones educativas, nos pintan un cuadro donde pocas cosas son lo que eran hace escasamente cinco o diez años y donde tenemos la impresión de que otras muchas van también a cambiar.

Con esa perspectiva comparativa que antes anunciaba, me ha parecido interesante presentar escuetamente la situación de las titulaciones educativas en la enseñanza superior de diversos países europeos para intentar establecer cuáles son las tendencias que se aprecian, a qué parecen responder, e incluso qué pistas de futuro nos aparecen anunciadas.

\subsection{Las titulaciones educativas en Francia}

Francia ha establecido claramente el sistema L-M-D (Licence-MasterDoctorat) en todas las universidades con lo que la estructura queda como en el siguiente cuadro en el que en la izquierda aparece claramente la nueva estructura mientras en la derecha observamos denominaciones aún existentes y correspondientes a la estructura anterior:

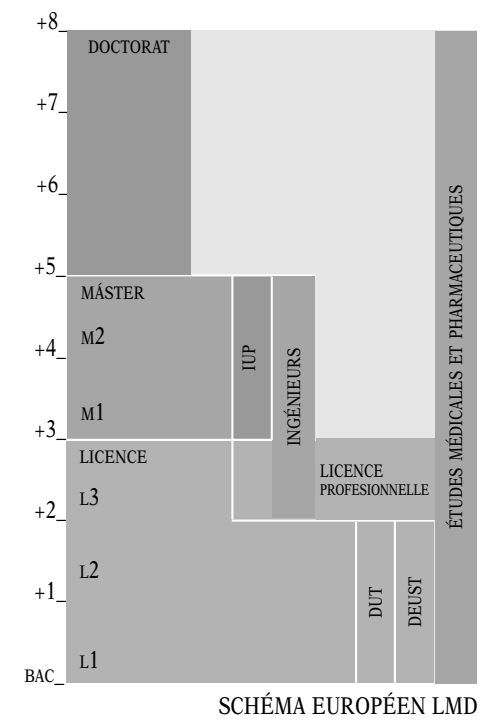

Como se observa los tres años de la Licence (Grado) dejan paso a los dos del Máster, que en la mayor parte de las universidades se diseñan de forma independiente (M1 y M2) y en algunas de ellas se plantean desde dos itinerarios, máster profesional y máster de investigación, que a su vez dará acceso al doctorado, realizado en algunos casos en las mismas Facultades y en otras en las Escuelas Doctorales que han sido creadas en varias universidades. 
Si en la estructura hay una gran homogeneidad, no es así en cuanto a las denominaciones y al diseño curricular de las titulaciones donde las universidades tienen bastante libertad para definirlo, en el caso de los estudios pedagógicos son frecuentes en el nivel del grado, las denominaciones: "Licence en Sciences de l'Education" o "Licence en Sciences de l'éducation et de la Formation" o "Licence en Sciences Humaines" (en la que una de las modalidades será Ciencias de la Educación), aunque también existen otras denominaciones mucho más concretas como "Métiers de la médiation sociale", "Consultant rurale", etc. A título de ejemplo se ofrece el diseño curricular correspendiente a la Universidad de Limoges, Dept. Sciences de l'Education:

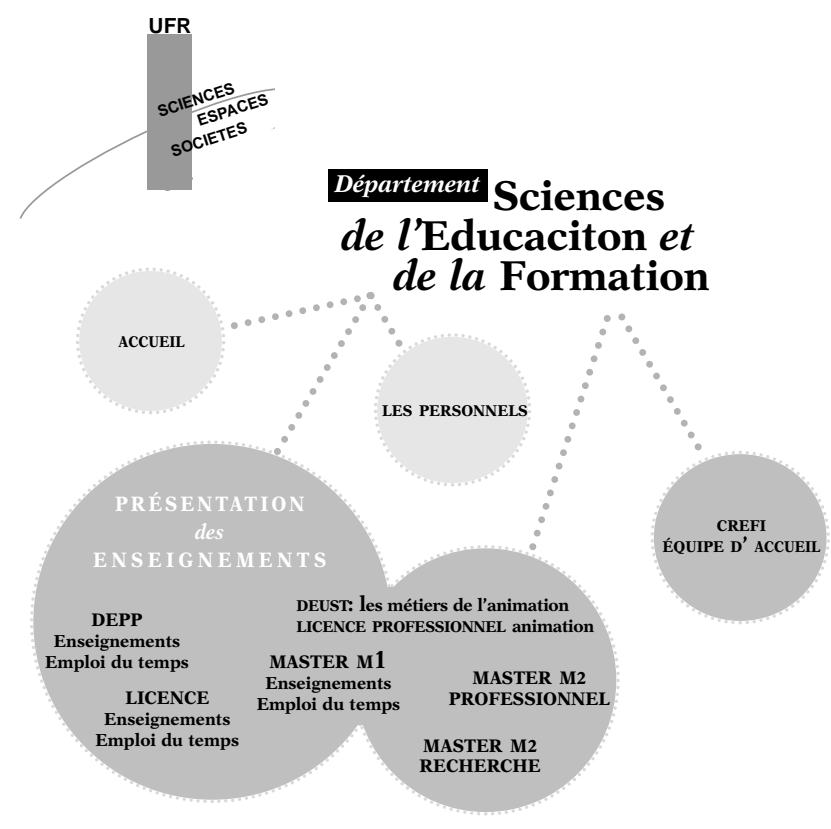

Podemos apreciar la presencia de la estructura L-M-D con la Licence y el Master en sus niveles 1 y 2, al tiempo que se mantienen estructuras antiguas, hoy reconvertidas, como un DEPP (Diploma de Enseñanzas Profesionales) o un DEUST(Diploma de Estudios Universitarios Científicos y Técnicos) en este caso en Animación Sociocultural, o una Licence Profesional. Estos diplomas se han mantenido en el sistema actual, aun cuando su reconversión al sistema L-M-D no esté, en algunos casos, totalmente terminada.

Si las titulaciones correspondientes a nuestra Pedagogía o Psicopedagogía quedan, en las universidades francesas en una estructura muy definida, las correspondientes a nuestra Educación Social, se sitúan en su mayoría fuera de esta estructura, pues sus características fundamentales serían:

a) Situarse a nivel de educación superior pero fuera de la enseñanza universitaria, aun cuando con algunas excepciones ${ }^{2}$. 
b) Desarrollarse preferentemente en centros privados dependientes del sector asociativo, con financiación pública parcial, aunque bastante importante.

c) Estar iniciando la transición hacia el sistema L-M-D, que algunos centros ya han acabado, y en consecuencia estar transformando sus antiguos Diplomas BAC+3 en "Licences professionnelles".

d) Mantener una importante diversidad de oferta de titulaciones, en la dualidad habitual en los modelos francófonos en los que el área de la Educación Especializada y el de la Animación Sociocultural quedan muy separados.

e) Establecer puentes con universidades francesas para asegurarse algún tipo de "paraguas" universitario para sus titulaciones y especialmente que sus estudiantes puedan seguir estudios accediendo directamente al nivel de Máster.

f) Plantear sus estudios tanto en la modalidad de "dedicación a tiempo completo" como en la de "promoción social" que implica unos horarios menos intensos, un diseño curricular un poco más largo y la equivalencia de ciertas partes del currículo por la experiencia profesional.

g) Mantener una fuerte vinculación con la práctica profesional, de manera que la mayor parte del tiempo de estudios se pasa en la práctica profesional, a partir de la cual se desarrollan los módulos teóricos.

Dentro de este panorama, el Diploma de Educación Especializada, es posiblemente el que más se acerca a la titulación española de Educación Social, aun cuando se especializa fundamentalmente en los ámbitos de la marginación, justicia juvenil, exclusión social y menores.

En ese panorama, la mayor parte de las titulaciones correspondientes a la Animación Sociocultural, algunas de ellas de bastante prestigio como el DEFA ${ }^{3}$ o el BEATEP $^{4}$ se mantienen con solo fuera de la enseñanza universitaria, sino que en la mayoría de los casos exigen una determinada experiencia profesional para poder acceder a las mismas.

\subsection{Las titulaciones de educación en Gran Bretaña}

La situación de la universidad británica es sin duda una de las que presentan mayor diversidad, tanto en lo que respecta a la denominación y contenidos de las titulaciones como en lo referente a su estructura. Tomemos algunos ejemplos: 
a) Kingston University, en el entorno de Londres nos presenta la clásica división de la Universidad británica entre "Undergraduates studies" y "Postgraduate Studies" que nos llevan al planteamiento británico del Grado y Máster. En su área de educación, encontramos a nivel de "Undergraduate" los siguientes títulos:

- Early Years: Education and Childcare BA(Hons) top-up

- Early Years: Senior Practitioner Foundation Degree

- Early Years: Teaching BA(Hons) top-up

- Primary Teaching leading to Qualified Teacher Status BA(Hons)

- Primary Teaching leading to Qualified Teacher Status PGCE

- Secondary Teaching leading to Qualified Teacher Status PGCE

b) La Oxford University ofrece por su parte los siguientes Másteres:

- Postgraduate Certificate in Education (PGCE)

- Master of Science Degree (MSc) in Educational Research Methodology

- Master of Science Degree (MSc) in Educational Studies (Comparative and International Education)

- Master of Science Degree (MSc) in Educational Studies (Higher Education)

- Master of Science Degree (MSc) in Educational Studies (E-Learning)

- Master of Science Degree (MSc) in Applied Linguistics and Second Language Acquisition

- Research degrees in Educational Studies

c) La University of Dundee (Escocia), nos ofrece por su parte las siguientes titulaciones en la "School of Education, Social Work and Community Education" cuyo título ya nos da una idea del planteamiento integrador de titulaciones que en nuestro caso distinguiríamos claramente como pertenecientes a los sectores de formación del profesorado, psicología, trabajo social y educación social, mientras que allí se plantean con bastantes aspectos comunes entre ellas:

En la línea de "Education" aparecen:

- BEd (Hons) Primary

- PGDE Primary

- PGDE Secondary

- Teaching Qualification (Further Education) TQFE (Postgraduate)

- Teaching Qualification (Further Education) TQFE (Undergraduate)

- Certificate in Teaching in Higher Education (CertTHE)

- M.Sc. in Educational Psychology

En la línea de "Community Education", encontamos:

- BA/BA (Hons) Community Education (Learning \& Development)

- Postgraduate Diploma in Community Education

Completando esta muestra con el Institute of Education de la University of London, que una muestra más diversa que las anteriores universidades, presenta los siguientes grados: 
- Diploma in Psychology

- Diploma in Special and Inclusive Education (Disabilities of Sigth)

- Diploma in Minority Ethnic Achievement

- Diploma in Professional Studies

- Diploma in Special and Inclusive Education

Así como los siguientes Másteres:

- Early Years Education

- Primary Education (policy and practice)

- Sociology of Childhood Education and Children's Rights

- Special Education (Inclusion and Disability Studies)

- Comparative Education

- Higher and Professional Education

- Teaching and Learning in Higher and Professional Education

- Higher Education Management

- Teaching

- A Teaching in the Post-Compulsory Sector

- Curriculum, Pedagogy and Assessment

- Inclusive Education

- Education(Citizenship/History/Religious Education), entre otros.

La estructura temporal de los "undergraduates" suele ser de tres o cuatro años y la de los másteres de uno o dos años. En ocasiones existe en las universidades un primer curso de estudios generales con lo cual la diversidad de estructuras temporales juntando grados y másteres es bastante grande, encontrando pues estructuras de: $3+1,3+2,4+1,1+3+1,1+2+1+1, \ldots$

Respecto a la orientación curricular, podemos observar claramente que los grados están enfocados hacia la docencia en educación infantil, primaria o secundaria y educación especial, apareciendo también la orientación de educación comunitaria, mientras que encontramos una diversidad de másteres muy grande, especialmente en las universidades grandes. Estos másteres se presentan en ocasiones como continuación y especialización de los grados, mientras que en otras sirven de inicio a la especialización a áreas pedagógicas determinadas.

Los curricula de los grados suelen estar formados de módulos bastante genéricos y poco especializados, como puede observarse en el ejemplo que se muestra correspondiente a la Universidad de Dundee, en el Degree of "Community Education": 
Programme Content

\begin{tabular}{|c|c|c|c|}
\hline & \multicolumn{3}{|c|}{3 Year Honours Degree with Professional Endorsement } \\
\hline Level 1 & Level 2 & Level 3 & Level 4 \\
\hline $\begin{array}{l}\text { - Personal and Professional } \\
\text { Development (1) (Faculty } \\
\text { Module) } \\
\text { - Professions in Context } \\
\text { (Faculty Module) } \\
\text { - Learning and Development: } \\
\text { Policies and Practice } \\
\text { - Working with People and } \\
\text { organisations (1) } \\
\text { - Practice (double module) } \\
\text { Total: } 6 \text { Modules }\end{array}$ & $\begin{array}{l}\text { - Personal and Professional } \\
\text { Development (2) } \\
\text { - Working with People and } \\
\text { organisations (2) } \\
\text { - Elective Modules choose } 2 \\
\text { from: } \\
\text { - Adult Education Studies } \\
\text { (1) } \\
\text { - Communication and } \\
\text { Information Technology } \\
\text { and Community Learning } \\
\text { - Community Arts } \\
\text { - Community Studies (1) } \\
\text { - Youth Education Studies } \\
\text { (1) } \\
\text { - Practice (double module) } \\
\text { Total: } 6 \text { Modules }\end{array}$ & $\begin{array}{l}\text { - Practice (double module) } \\
\text { - An Inquiry Focus on } \\
\text { Contemporary Issues in } \\
\text { Learning and Development } \\
\text { - Elective Module choose } 1 \\
\text { from: } \\
\text { - Adult Education Studies } \\
\text { (2) } \\
\text { - Community Studies (2) } \\
\text { - Equality Studies (1) } \\
\text { - Volunteering and the } \\
\text { Voluntary Sector } \\
\text { - Youth Education Studies } \\
\text { (2) } \\
\text { = BA Community } \\
\text { Education with } \\
\text { Professional Endorsement } \\
\text { Total: } 4 \text { Modules }\end{array}$ & $\begin{array}{l}\text { - An Inquiry Focus on } \\
\text { Sociology of Community } \\
\text { Linked with Innovation } \\
\text { Practices in Learning and } \\
\text { Development (double module) } \\
\text { - Elective Module choose } 2 \\
\text { from: } \\
\text { - Adult Education Studies } \\
\text { (3) } \\
\text { - Community Studies (3) } \\
\text { - Equality Studies (2) } \\
\text { - Youth Education Studies } \\
\quad \text { (3) } \\
\text { = BA Community } \\
\text { Education with } \\
\text { Professional Endorsement } \\
\text { Total: } 4 \text { Modules }\end{array}$ \\
\hline
\end{tabular}

con asignaturas bastante genéricas y que continúan a lo largo de varios cursos. Por el contrario en los Másteres nos encontramos con niveles de especialización bastante elevados.

Por lo que respecta a las denominaciones de los títulos, la universidad británica se distingue por una cierta diversidad, menor que la encontrada en la universidad francesa, pero en cualquier caso predominan los títulos como "Bachelor of Arts" y "Bachelor of Education", con una mayor diversidad en las denominaciones de los grados.

\subsection{Las titulaciones de educación en Italia}

La reforma de las titulaciones universitarias italianas ha sido realizada, al menos desde el punto de vista estructural a partir del curso 2001-2002. Como ocurre en la mayoría de países europeos se ha optado por una estructura 3+2, transformando las antiguas "laureas" cuatrienales en una "laurea" trienal y una "laurea specialistica" de dos años de duración. Junto a esto, algunas universidades han implementado también "másteres" que pueden durar uno o dos años siendo de primer o segundo nivel. Ambas vías dan acceso a un doctorado de investigación, quedando así el esquema:

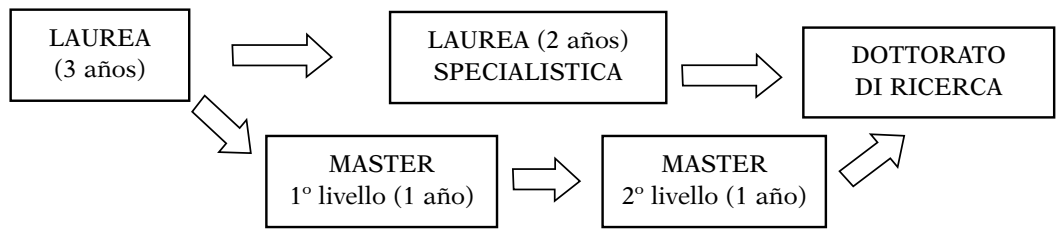


Por el contrario la reforma metodológica apenas está empezando. En este mismo curso 2007-2008, se está procediendo al cambio de la medida en horas por la medida en créditos ECTS, mientras que las reformas estrictamente metodológicas concerniendo al desarrollo de las clases y de la evaluación apenas se han iniciado en algunos centros.

En la mayoría de las universidades aún conviven planes antiguos y nuevos de las titulaciones educativas, que de manera similar a la situación francesa, mantienen una gran diversidad aun cuando hay varias denominaciones que existen de forma mayoritaria en casi todas las universidades, especialmente la que se refiere a la formación del maestro de primaria. Lo que si es generalizado es que el conjunto de las titulaciones educativas se realizan en el seno de la universidad, tanto las referidas al campo de las ciencias de la educación, -con mayor semejanza a la pedagogía española-, como las referentes a la formación de maestros y a la educación social, cuyo titulado es denominado de manera preferente en el conjunto de Italia, "educador profesional". plos:

Por lo que se refiere a las titulaciones existentes, veamos algunos ejem-

a) En la Università di Salento, con sede en la ciudad de Lecce, encontramos en la Facultad de "Scienze della Formazione" cuatro laureas trienales

- Pedagogia dell'Infanzia

- Esperti della Formazione

- Educatori Professionali

- Scienze Umane e Morali

Una "laurea specialistica" de dos años: "Scienze Pedagogiche" y dos másteres de un año de duración:

- Máster I (primer nivel) "Animazione musicale e musicoterapia";

- MásterII (segundo nivel) "Professionisti nella relazione d'aiuto per il disagio, la tossicodipendenza e la disabilità";

así como un Máster de nivel I y II al mismo tiempo de dos años de duración y centrado en: "Pedagogia interculturale e dimensione europea dell'educazione".

b) Por su parte la Universidad de Bolonia ofrece cuatro laureas trienales: "Educatore di nido e di comunita' infantile, Educatore sociale. Formatore (promozione e sviluppo risorse umane) y Operatore culturale/esperto in scienze dell'educazione", mientras que sigue manteniendo una laurea con el viejo esquema cuatrienal "Scienze della formazione primaria". En cuanto a los Másteres, ofrece una gran variedad en la rama humanística siendo los más cercanos al mundo de las ciencias de la educación, los siguientes: La dimensione educativa della cooperazione internazionale, Comunicare con gli adolescenti: la pre- 
venzione del disagio giovanile, Pluralismo religioso: identità, conflitti, dialogo tra fedi.

c) La universidad "Roma-3" nos ofrece en su Facultad de Scienze della Formazione" las siguientes laureas trienales:

- Discipline del Servizio Sociale ad Indirizzo Formativo Europeo

- Educatore Professionale di Comunità

- Formazione e Sviluppo delle Risorse Umane

- Scienze dell'Educazione

- Scienze Sociali della Conoscenza e della Formazione

Y las siguientes laureas "magistrale" (specialistica): Discipline del Servizio Sociale ad Indirizzo Formativo Europeo, Educatore Professionale di Comunità, Formazione e Sviluppo delle Risorse Umane, Scienze dell'Educazione, Scienze Sociali della Conoscenza e della Formazione, algunas de las cuales dividiéndose a su vez en diversas especialidades, como por ejemplo la laurea en "Scienze dell'Educazione" que contempla las siguientes modalidades:

- Esperti di Processi Psico-educativi e Relazionali

- Formazione Interculturale e Socio - Educativa

- Operatori Pedagogici ed Esperti di Processi di Comunicazione Didattica

Si nos fijamos en el diseño curricular de las titulaciones más cercanas a las nuestras ("Scienze dell'educazione" y "educatore professionale/sociale"), que en general son bastante semejantes en la mayoría de las universidades, nos encontramos con el siguiente planteamiento:

Laurea in "EDUCATORE SOCIALE". UNIVERSITÀ DI BOLOGNA.

Listado de materias (todas ellas de 5 créditos)

- 00045 - Antropologia Culturale

- 06992 - Antropologia Filosofica

. 43312 - Didattica dell'Integrazione

- 08824 - Didattica Generale

- 42972 - Diritto dei Servizi Sociali

- 56241 - Filosofia Teoretica

- 57663 - Il Prodotto Culturale

- 00639 - Lingua Francese

- 00639 - Lingua Frencese (G.A)

- 00644 - Lingua Inglese

- 00651 - Lingua Spagonla

- 00955 - Metodologia del Lavoro di Gruppo

- 54510 - Metologie Qualitative e Quantitative della Ricerca Sociale

- 54771 - Modelli e Strumenti del Gioco e del Videogioco

- 54767 - Modelli e Strumenti di Ricerca Educativa e Riabilitativa

. 41092 - Pedagogia della Comunicazione 
- 01351 - Pedagogía della Marginalita' e della Devianza

- 54769 - Pedagogia della Narrazione

- 56240 - Pedagogia Generale

- 15579 - Pedagogia Interculturale

- 04517 - Pedagogia Sociale

- 00797 - Pedagogia Speciale

- 04538 - Poetica e Retorica

- 04875 - Psicologia dei Gruppi

- 54768 - Psicologia del Ciclo di Vita

- 07253 - Psicologia dell'Educazione

- 03079 - Psicologia dello Sviluppo

- 56238 - Psicologia Generale (7CFU)

- 00870 - Psicologia Sociale

- 12612 - Psicopatologia dello Sviluppo

- 04491 - Sociologia dei Processi Culturali

- 12078 - Sociologia della Devianza

- 02515 - Sociologia della Famiglia

- 54770 - Sociologia della Prevenzione

- 56239 - Sociologia Generale (7CFU)

- 00932 - Storia Contemporanea

- 61895 - Storia dei Genocidi Internazionali

- 56242 - Storia della Filosofia

- 48583 - Storia delle Culture

- 16764 - Storia dell'Educazione

- 11379 - Storia Medievale

- 00929 - Storia Moderna

- 61631 - Tecniche di Preparazione dei Materiali

- 11348 - Tecnologie dell'Educazione

- 48584 - Teorie della Differenza Sessuale

- 48587 - Teorie e Strumenti dell' Intelligenza Artificiale

- 01369 - Teorie e Tecniche della Dinamica di Gruppo Tirocinio (Prácticas)

Por lo que respecta a la titulación más cerca de "Pedagogía", un ejemplo sería:

- Laurea in "SCIENZE DELL'EDUCAZIONE" - UNIVERSITÀ DI ROMA III Modalidad: Operatori Pedagogici ed Esperti di Processi di Comunicazione Didattica. 
Primmo anno

Linguistica Generale

1 disciplina a scelta tra: (a elegir entre)

- Didattica Generale (SSD M-PED/03) (CFU 4)

- Tecnologie dell'Istruzione e dell'Apprendimento (SSD M-PED/03) (CFU 4)

1 disciplina a scelta tra:

- Docimologia (SSD M-PED/04) (CFU 4)

- Pedagogia Sperimentale (SSD M-PED/04) (CFU 4)

\section{1 disciplina a scelta tra:}

- Metodologia della Ricerca Pedagogica (SSD M-PED/01) (CFU 4)

- Pedagogia Generale (SSD M-PED/01) (CFU 4)

\section{1 disciplina a scelta tra:}

- Storia della Pedagogia (SSD M-PED/02) (CFU 4)

- Storia della Scuola e delle Istituzioni Educative (SSD M-PED/02) (CFU 4)

Psicologia Generale

Storia Contemporanea I

Storia Moderna I

Storia de la Filosofia

\section{1 disciplina a scelta tra:}

- Pedagogia Interculturale (SSD M-PED/01) (CFU 4)

- Storia dell'Educazione (SSD M-PED/02) (CFU 4)

\section{CFU a scelta tra:}

- Laboratorio di Lingua e Traduzione Spagnola (SSD L-LIN/07) (CFU 2)

- Lingua e Traduzione Spagnola (i anno) (SSD L-LIN/07) (CFU 4)

- Lingua Francese I (SSD L-LIN/04) (CFU 6)

- Lingua Inglese (SSD L-LIN/12) (CFU 6)

Abillità Informatiche

\section{1 disciplina a scelta tra:}

- Laboratorio di Educazione Estetica (SSD M-PED/01) (CFU 2)

- Laboratorio di Pedagogia Interculturale (SSD M-PED/01) (CFU 2)

- Seminario - Lettura e Analisi dei Classici della Filosofia (SSD M-FIL/06) (CFU 2)

- Seminario - Lettura e Analisi dei Classici della Pedagogia (SSD M-PED/02) (CFU 2)

- Seminario - Oralità e Scrittura nell'Educazione Occidentale (SSD M-FIL/06) (CFU 2)

\section{1 disciplina a scelta tra:}

- Sociologia (SSD SPS/07) (CFU 4)

- Sociologia Generale (SSD SPS/07) (CFU 4)

- Sociologia I (SSD SPS/07) (CFU 4) 


\section{CFU a scelta tra:}

- Lingua Francese II (SSD ND) (CFU 4)

- Lingua Inglese (SSD L-LIN/12) (CFU 4)

- Lingua Spagnola 2 Sem. (SSD L-LIN/07) (CFU 4)

1 disciplina a scelta tra:

- Filosofia dell'Educazione (SSD M-PED/01) (CFU 4)

- Pedagogia della Comunicazione (SSD M-PED/01) (CFU 4)

Psicologia dello Sviluppo

1 disciplina a scelta tra:

- Sociologia dell'Educazione (sSD SPS/08) (CFU 4)

- Sociologia delle Comunicazioni di Massa (SSD SPS/08) (CFU 4)

Storia della Filosofia Antica

\section{1 disciplina a scelta tra:}

- Igiene Scolastica (SSD MED/42) (CFU 4)

- Psichiatra (SSD MED/39) (CFU 4)

\section{1 disciplina a scelta tra:}

- Psicologia della Salute (SSD M-PSI/08) (CFU 4)

- Psicopatologia Generale (SSD MED/25) (CFU 4)

\section{1 disciplina a scelta tra:}

- Educazione Comparada (sSD M-PeD/02) (CFu 4)

- Storia della Letteratura per l'Infanzia (SSD M-PED/02) (CFU 4)

\section{1 disciplina a scelta tra:}

- Pedagogia della Marginalità e della Devianza Minorile (SSD M-PED/03) (CFU 4)

- Pedagogia Speciale (sSD M-PED/03) (CFU 4)

\section{1 disciplina a scelta tra:}

- Didattica Speciale (SSD M-PED/03) (CFU 4)

- Docimologia (SSD M-PED/04) (CFu 4)

- Metodologia e Tecnica del Gioco e dell'Animazione (sSD M-PeD/03) (CFu 4)

\section{1 disciplina a scelta tra:}

- Instituzione di Diritto Pubblico e Legislazione Scolastica (SSD IUS/09) (CFU 4)

- Legislazione Minorile (SSD IUS/09) (CFU 4)

\section{2 disciplina a scelta tra:}

- Laboratorio Modelli Comparati di Educazione degli Adulti (sSD M-PED/01) (CFu 2)

- Laboratorio di Pedagogia Speciale "Disabilità e Counselling Educativo (SSD M-PED/03) (CFU 2)

- Laboratorio di Pedagogia Teatrale ed Economia dell'Apprendimiento (sSD M-PED/01) (CFU 2)

- Laboratorio di Scrittura Digitale (SSD M-PED/03) (CFU 2) 


\section{LISTADO DE MATERIAS}

\section{1 disciplina a scelta tra:}

- Psicologia dell'Attaccamento nel Ciclo di Vita (sSD M-PSI/04) (CFu 4)

- Psicologia Sociale (SSD M-PSI/05) (CFU 4)

\section{1 disciplina a scelta tra:}

- Storia Contemporanea II (sSD M-STo/04) (CFu 4)

- Storia Moderna II (SSD M-STO/02) (CFU 4)

1 disciplina a scelta tra:

- Antropologia Culturale (SSD M-DEA/01) (CFu 4)

- Antropologia Sociale (SSD M-DEA/01) (CFU 4)

1 disciplina a scelta tra:

- Sociologia dei Processi Culturali (sSD SPS/08) (CFU 4)

- Sociologia della Famiglia (sSD SPS/08) (CFU 4)

Psicologia Clinica

1 disciplina a scelta tra:

- Educazione degli Adulti (SSD M-PED/01) (CFU 4)

- Educazione Psicomotoria (SSD M-PED/01) (CFU 4)

- Metodologia dell'Educazione Interculturale (SSD M-PED/01) (CFU 4)

1 disciplina a scelta tra:

- Economia dell'Istruzione e dell'Apprendimento (SSD SECS-P/06) (CFU 4)

- Statistica (SSD SECS-S/01) (CFU 4)

\section{1 disciplina a scelta tra:}

- Laboratorio di Cinematografia (SSD L-ART/06) (CFU 2)

- Laboratorio di Metodologia dell'Educazione Musicale (SSD L-ART/06) (CFU 2)

Bibliografia e Biblioteconomia

1 disciplina a scelta tra:

- Musicologia e Storia della Musica (SSD L-ART/07) (CFU 4)

- Semmiologia del Cinema e degli Audiovisivi (SSD L-ART/06) (CFU 4)

Analizando ambos ejemplos observamos varias características comunes a los grados italianos en el ámbito de la educación:

- Los curricula son bastante genéricos con materias comunes a otros ámbitos como humanidades, lingüística, ciencias sociales o psicología.

- En general no tienen especialidades, (Roma III sería una excepción) dentro de las "laurea" (grados) aun cuando aparecen en los Masters y las "lauree specialistiche". 
- El diseño curricular cuenta con un elevado número de asignaturas (1215 por curso), habitualmente semestrales y con un creditaje reducido (45 créditos).

- No siempre aparecen períodos de prácticas integradas en el plan de estudios.

- Es habitual la presencia de una o dos lenguas extranjeras en el currículo, pero su desarrollo no suele pasar de un cuatrimestre.

- El diseño de los grados en la universidad italiana está más cercano a un planteamiento universitario que profesional, no soliendo disponer de materias referidas a instrumentos de intervención profesional.

\subsection{Las titulaciones de educación en Bélgica}

Los estudios pedagógicos en las universidades belgas tienen una larga tradición pues son creados a partir de una ley de 1890 y casi siempre han mantenido una cercanía hacia la psicología. Lo que hace que en muchas facultades ambas titulaciones (Psicología y Ciencias de la Educación) aparezcan en la misma facultad.

Como en el caso de los estudios franceses, la enseñanza superior belga cuenta con casi la mitad de sus formaciones realizándose fuera de las universidades. En los últimos diez años se procedió a una concentración de estas titulaciones con la creación de las "Hautes Écoles". En ellas se integraron titulaciones referidas a la Educación Social y a la formación del profesorado de Primaria y Secundaria. Con esto se reproduce el modelo que antes presentábamos referido a Francia, con unas titulaciones con una carga conceptual más elevada desarrollándose en el seno de la universidad y por otra parte, otro grupo de titulaciones mucho más profesionalizadoras, pero con menor nivel conceptual, que se desarrollan en las Escuelas Superiores.

Hay que indicar también que la reforma de la estructura universitaria se terminó hace dos años y estableció el sistema 3+2 (Bachelier + Master) que dará posteriormente acceso a los estudios de doctorado. Los estudios de grado en ciencias de la Educación y Psicología se desarrollan a lo largo de tres años y comportan 180 créditos de los cuales 150 son comunes a las diversas especialidades que puedan existir. Estas especialidades se desarrollan durante el tercer curso. En la Universidad de Lovaina (Valonia), por ejemplo, se plantean tres: Ciencias Psicológicas, Ciencias de la Educación y Logopedia. A título de ejemplo, presento el diseño curricular de Ciencias de la Educación: 
Première année

- Tronc commun

- Question de sciences religieuses (a elegir entre)

- Questions de sciences religieuses: lectures bibliques [15h] (2 crédits)

- Questions de sciences religieuses: Christianisme et questions de sens [15h] (2 cr)

- Questions de sciences religieuses: questions d'éthique [15h] (2 crédits)

- Fondements socioculturels du comportement

- Notions de philosophie [30h] (3 crédits)

- Introduction à l'épistémologie [30h] (3 crédits)

- Sociologie [30h] (3 crédits)

- Introduction aux sciences du langage [30h] (3 crédits)

- Fondements biologiques du comportement

- Biologie générale et génétique [37.5h] (3 crédits)

- Physiologie humaine [22.5h] (3 crédits)

- Neurophysiologie [37.5h $+4 \mathrm{~h}]$ (3 crédits)

- Ethologie et psychologie comparée [30h] (3 crédits)

- Psychologie, éducation, développement

- Psychologie générale I [90h] (8 crédits)

- Psychologie générale II [45h] (4 crédits)

- Sciences de l'éducation I [30h + 15h] (5 crédits)

- Psychologie du développement [75h] (8 crédits)

- Pratiques et méthodes

- Monitorat de psychologie $[0 \mathrm{~h}+37.5 \mathrm{~h}]$ (3 crédits)

- Méthodes de l'observation [0h $+30 \mathrm{~h}]$ (4 crédits)

- Langues: Anglais - $2 \mathrm{cr}$.

Deuxième année

- Tronc commun

- Fondements biologiques du comportement

- Psychophysiologie et neuropsychologie [60h] (5 crédits)

- Psychologie, éducation, développement

- Sciences de l'éducation II $[60 \mathrm{~h}+15 \mathrm{~h}]$ (5 crédits)

- Bien-être et santé

- Psychologie de la personnalité et de l'individu [30h $+5 \mathrm{~h}]$ (3 crédits)

- Psychologie et Santé $[60 \mathrm{~h}+10 \mathrm{~h}]$ (6 crédits)

- Travail, organisation et société

- Psychologie sociale, du travail et des organisations [90h + 15h] (9 crédits)

- Pratiques et méthodes

- Travaux pratiques $[0 \mathrm{~h}+30 \mathrm{~h}](2$ crédits $)$ 
- Méthodologie de l'expérimentation [30h] (3 crédits)

- Statistique appliquée à la psychologie I $[30 \mathrm{~h}+20 \mathrm{~h}](5$ crédits $)$

- Psychométrie (y compris édumétrie) $[30 \mathrm{~h}+10 \mathrm{~h}]$ (4 crédits)

- Langues: Anglais-Compréhension de textes de psychologie [30h] (3 crédits)

Une mineure à choisir: el estudiante debe elegir $15 \mathrm{cr}$. De una especialidad de otras titulaciones de la UCL o bien de materias de profundización de su titulación.

Troisième année

Une majeure à choisir (una modalidad que condicionará su acceso al Máster). Se ofrecen tres modalidades: ciencias psicológicas, ciencias de la educación y logopedia. He aquí el currículo de la correspondiente a la segunda de ellas.

- Majeure (modalidad) en sciences de l'éducation

- Sciences psychologiques - [30h] (4 crédits)

- Psychologie des ressources humaines [30h] (4 crédits)

- Neuropsychologie de l'enfant [30h] (4 crédits)

- Psychologie des situations de handicap et psychoéducation [30h] (4 crédits)

- Développement, milieu et culture [30h] (4 crédits)

- Entretien psychologique $[15 \mathrm{~h}+30 \mathrm{~h}]$ (3 crédits)

- Approches disciplinaires en éducation et en formation

- Philosophie de l'éducation et de la formation [60h] (5 crédits)

- Sociologie de l'éducation et de la formation [45h $+15 \mathrm{~h}]$ (5 crédits)

- Economie de l'éducation et de la formation [45h] (5 crédits)

- Approche historique de l'éducation et de la formation [30h] (5 crédits)

- Observation participante de pratiques professionnelles

- Projet, avec séminaire d'accompagnement $[15 \mathrm{~h}+40 \mathrm{~h}]$ (11 crédits)

Cada uno de los "bacheliers" dan acceso directo a un Máster. En este caso, la modalidad de Ciencias de la Educación de este grado da acceso directo al "Máster en Ciencias de la Familia y de la Sexualidad", pudiendo también acceder a otros Másteres con algunos complementos de formación.

Por su parte, la Universidad de Gante (Flandes), con la misma estructura de "bachelier-master" ofrece el "Bachelier en Sciencies Psychologiques et de l'Education" que presenta las siguientes modalidades:

- Bachelor of Psychology

- Bachelor of Psychology - Espec.: Clinical Psychology

- Bachelor of Psychology - Espec.: Personnel Management and Industrial Psychology

- Bachelor of Psychology-Espec.: Theoretical and Experimental Psychology 
- Bachelor of Educational Sciences

- Bachelor of Educational Sciences - Espec.: Foundations and Practice of Education

- Bachelor of Educational Sciences - Espec.: Special Education, Disability Studies and Behavioral Disorders

- Bachelor of Educational Sciences - Espec.: Social Work and Social Welfare Studies

Y los siguientes programas de Másteres, a los que se accede en algunos casos con estudios complementarios:

- Master of Psychology - Main Subject: Clinical Psychology

- Master of Psychology - Main Subject: Theoretical and Experimental Psychology

- Master of Psychology - Main Subject: Personnel Management and Industrial Psychology

- Master of Psychology - Main Subject: Teacher Education and Training

- Master of Educational Sciences - Main Subject: Foundations, Theory and Study of Education

- Master of Educational Sciences - Main Subject: Special Education, Disability Studies and Behavioral Disorders

- Master of Social Work and Social Welfare Studies

Por contra los estudios profesionales como la Educación Social o la formación del profesorado se realizan como antes se indicaba en las "Hautes Ecoles" y durante bastante tiempo han estado muy alejados de la universidad. Con el establecimiento de la nueva estructura, se están empezando a desarrollar ciertas pasarelas que les permiten el acceso a los Másteres universitarios. Tomando un ejemplo, se presenta el plan de estudios del "Centre de Formation d'Educateurs liegeois" de la Haute Ecole ISELL:

\section{Número de horas por curso}

\section{Formation générale}

Audiovisuel et multimédias

Expression orale et écrite

Philosophie

Questions religieuses

Formation à la neutralité $\mathbf{1}^{\text {ere }} \quad 2^{\text {éme }} \quad 3^{\text {éme }}$

$\begin{array}{ccc}50 & / & / \\ 25 & 25 & / \\ 25 & 25 & 25 \\ 35 & / & / \\ / & / & 20\end{array}$

2. Formation sociologique, économique et juridique

Déontologie

/ $\quad$ / 25

Droit

25 / /

Économie politique

Législation enfance et handicap
/ 25 /

/ $25 \quad /$ 
Organisation des institutions socio-éducatives et culturelles

$\begin{array}{ccc}/ & 30 & 45 \\ 50 & 25 & / \\ / & 25 & / \\ 25 & / & /\end{array}$

Sociologie

Politiques sociales

\section{3. Éducation à la santé}

Anatomie, pshysiologie, biologie

Éducation à la santé

/ 25

Neuropsychopathologie

\section{Techniques et méthodologies de la profession}

Activités physiques et sportives:

éducation physique et psychomotrice

Initiation à la sécurité et à l'entretien de matériel:

espace, environnement, éducation

Techniques artistiques (plastiques, musicales):

animation et techniques créatives

didactique de l'expression (plastique, musicale, corporelle)

techniques créatives

Techniques d'animation:

méthodologie espéciale: travail de groupe

séminaire de pratique professionnelle

$70 \quad 50 \quad /$

Technique d'expression orale et écrite:

didactique de l'expression

langage et communication

$25 \quad 1$

pratiques théâtrales

techniques de l'entretien individuel

$1-1+257$

\section{Formation psychologique et pédagogique}

Ateliers de recherche pédagogique

$\begin{array}{ccc}/ & / & 50 \\ / & 25 & / \\ 50 & / & / \\ / & / & 25 \\ / & 25 & / \\ / & / & 50 \\ / & 50 & / \\ 75 & / & / \\ / & 25 & / \\ / & 25 & /\end{array}$

Facultad de Educación. UNED 
Histoire de l'éducation spécialissé

Modèles d'intervention en éducation spécialisée

Psychologie générale

$\begin{array}{ccc}25 & / & / \\ / & 25 & / \\ 30 & / & /\end{array}$

6. Activités d'intégration professionnelle

Compléments de formation

$10 \quad 10 \quad 10$

Travil de fin d'études (TFE)

/ / 30

Stages et accompagnement de la pratique professionnelle

La formación se desarrolla durante tres años y en líneas generales es semejante a la de otras escuelas de educadores especializados belgas, aun cuando hay bastantes singularidades, especialmente en cuanto al método de trabajo, ya que algunas escuelas trabajan prácticamente en alternancia con las prácticas que se llevan la mayor parte del currículo.

En resumen, en las titulaciones educativas de la enseñanza superior belga, asistimos al sistema dual de Universidades y Escuelas Superiores que es común a bastantes países del centro de Europa (Francia, Bélgica, Holanda, Dinamarca, Luxemburgo, Suiza, Alemania...). Las enseñanzas se han adaptado a la estructura $3+2$ de Bolonia y presentan en las universidades grados de carácter generalista con un fuerte tronco común, aun cuando se plantean especialidades o modalidades que condicionan los accesos a los Másteres. Estos, bastante más especializados, se desarrollan por $120 \mathrm{cr}$ y al igual que los grados suelen contemplar diversas modalidades de prácticas.

Las enseñanzas que se imparten en las Escuelas Superiores, en nuestro caso, las correspondientes a la Educación Social y a la formación del profesorado tienen un currículo bastante generalista que se especializa a partir de la explotación de los períodos de prácticas que suelen triplicar y cuadruplicar el número de horas de las prácticas integradas en las titulaciones españolas. Como ocurría en el sistema francés, los "bacheliers" realizados en las Universidades y en las Escuelas Superiores, están alejados, presentan claras diferencias curriculares y metodológicas y no suelen mantener contactos, aun cuando coinciden en un diseño de módulos breves (casi ninguno pasa de los 4-5 cr.) y un número importante de materias a lo largo de la titulación.

\subsection{Las titulaciones de educación en Alemania}

El sistema universitario alemán se ha caracterizado de lo largo de su historia por presentar unas titulaciones con una fuerte carga cognitiva y desarrolladas en un importante número de años. En la universidad alemana se está aplicando también la reforma del espacio europeo de educación superior y en consecuencia la transformación a una estructura $3+2$. No obstante el proceso transcurre con bastante lentitud y algunas universidades lo acaban de iniciar. 
La estructura de las universidades alemanas en cuanto a la composición de sus facultades difiere bastante entre sí, pero es habitual que la educación se trabaje junto a la psicología, la filosofía o las ciencias del comportamiento. Así por ejemplo, la Universidad de Hamburgo tiene una Facultad de Ciencias de la Educación, Psicología y Ciencias del Movimiento, cuya sección de educación se divide a su vez en cinco especialidades

- Educación Comparada, Internacional e Intercultural

- Pedagogía Escolar, Social, Compensatoria y Psicología Educativa

- Formación Profesional y Aprendizaje Permanente

- Didáctica de la Educación lingüística y estética

- Didáctica de la educación científico-matemática

La mayoría de los grados establecidos en la estructura de Bolonia se mueven entre 180-210 créditos y plantean asignaturas globales con una fuerte carga filosófica, sociológica y cultural, para desarrollar después másteres que en la mayoría de los casos aún no se han iniciado o mantienen las antiguas formaciones con duración entre cinco años y medio a seis años.

En los últimos diez-quince años se han creado unas cuantas universidades técnicas o de ciencias aplicadas como transformación de las antiguas Fachhochsule. Las formaciones sobre Educación Social/Trabajo Social (Bachelor-Studiengang Soziale Arbeit/Sozial Pedagogen), así como la Pedagogía Infantil (Bachelor-Studiengang Pädagogik der Kindheit) se están desarrollando en este tipo de centros. Con estructura reformada (habitualmente $3+2$ ), plantean un diseño curricular con materias bastante globales y unas prácticas de importancia mayor al diseño desarrollado en las universidades.

La integración de las Fachhochsule en la estructura universitaria ha facilitado el paso entre sus titulaciones y los Másteres Universitarios, aun cuando el sistema como se indicaba anteriormente se inicia en estos años y aún faltan unos cuantos para la correcta integración de ambas estructuras que en realidad actualmente ya forman una sola, pero con tradiciones y metodologías bien diferenciadas.

\section{CONCLUSIONES}

La descripción que se ha realizado de los estudios de educación en la enseñanza superior europea (universidades y enseñanza superior no universitaria) se ha limitado a la estructura, el diseño curricular, las características de los módulos y las relaciones entre las diversas etapas del sistema universitario, con algún apunte sobre los períodos de prácticas. No se ha entrado, y sería bien interesante, en el análisis de la metodología dominante en las titulaciones educativas y esto sería posiblemente el elemento más interesante para percibir hasta que punto se está produciendo un verdadero cambio en la enseñanza uni- 
versitaria. La dificultad es que es esto requeriría una investigación cualitativa basada fundamentalmente en fuentes primarias (observación de la realidad, entrevistas al profesorado y encuestas y foros con los estudiantes).

Con todo, el análisis realizado permite llegar a algunas conclusiones que en ciertos casos se plantean como una información interesante de cara a elaborar nuestros próximos planes de estudio. Destacaría las siguientes:

a) De los seis países analizados, cuatro de ellos (Alemania, Bélgica, Francia e Italia) han establecido claramente la estructura 3+2 (gradomáster) en sus enseñanzas universitarias, Gran Bretaña presenta una diversidad de estructuras, entre las que también existe la de $3+2$, junto con otras $(4+1,1+3+1)$ y España será el único país entre los estudiados donde se establecerá de manera generalizada la estructura 4+1. En el conjunto europeo, la estructura $3+2$ es claramente mayoritaria en este momento en el que la reforma de Bolonia, en su faceta estructural, está casi completamente desarrollada. Esto generará algunas dificultades a la movilidad de estudiantes españoles y planteará algunos inconvenientes con las equivalencias de los estudios para los que vengan del resto de Europa.

b) En tres de los países analizados, -Francia, Bélgica, Alemania-, existe una diferencia importante entre los estudios pedagógicos, realizados en la universidad y los correspondientes a la Educación Social que se sitúan en la enseñanza superior no universitaria. Esta no es la única diferencia, puesto que los estudios no universitarios explotan mucho más la relación práctica-teoría que en algunos casos se convierte en estudios en alternancia. Al mismo tiempo, sus módulos teóricos son breves y profesionalizadores, dejando bastante de lado la faceta investigadora.

c) España es el único caso donde la denominación de las titulaciones es, hasta ahora, muy homogénea, con tres o cuatro apelaciones que se distribuyen por todo el estado y están centralizadas en un catálogo de títulos. Bélgica lo plantea de manera semejante aunque existe un poco más de diversidad, especialmente si comparamos las universidades flamencas y valonas. En los demás países la diversidad de denominaciones de los títulos educativos es en general grande, en un abanico que nos llevaría desde Italia o Francia con títulos relativamente homogéneos aunque encontramos una quincena de apelaciones diversas, a Gran Bretaña donde cada universidad plantea sus títulos partiendo de una etiqueta común (Bachelor of Arts) que se concreta de formas muy diferentes.

d) Si la diversidad entre los grados de los diferentes países es grande, lo es mucho más entre los másteres que presentan una gran heterogeneidad de enfoques. En este caso es en Italia donde aparecen denomina- 
ciones más genéricas, mientras que en el resto de países las diferentes universidades establecen sus líneas de especialización. Desde ese punto de vista parece claro que la homologación de los niveles de estudio deberá hacerse en función de estructuras semejantes pero en función de contenidos. Es decir que un estudiante podrá haber cursado el mismo número de créditos ECTS, pero sin duda habrá trabajado aspectos bastante diversos. A la luz del análisis, el intento del Tuning de educación para elaborar un esquema de título común parece muy lejano en el área educativa.

e) En los grados, el contenido de los planes de estudio ofrece un gran atractivo por su gran diversidad. En general nos encontramos con diseños poco especializados en los que suelen aparecer materias referidas a las ciencias sociales, a las humanidades o a las lenguas extranjeras. Esto es especialmente remarcable en la universidad italiana, francesa, alemana y belga. Además en estas universidades los módulos son más bien cortos no soliendo pasar de 4-5 ECTS. Desde el punto de vista de las materias que forman el currículo, es sin duda la universidad italiana la que ofrece el modelo más "clásico" con bastantes materias comunes a otras titulaciones no estrictamente educativas. El tamaño menor de los módulos aparece en la universidad francesa o belga, con materias de dos créditos en muchos casos y un nivel más elevado de especialización.

f) Por el contrario la universidad británica presenta en los grados, módulos de larga duración (entre 10-30 créditos) que además se suceden en los diferentes cursos. Estos enfoques bastante globales permiten hacer planteamientos de continuidad y al mismo tiempo singularizar su contenido en función del sello de una facultad o un departamento.

g) Cuando las titulaciones pedagógicas y socioeducativas están insertas en la universidad (Italia, Gran Bretaña y parcialmente Alemania) sus diseños tienen un porcentaje importante de similitud y sus módulos se repiten en ambas titulaciones. Por el contrario cuando se trata de enseñanzas universitarias (los estudios pedagógicos) y no universitarias (los socioeducativos), sus curricula difieren bastante, tanto en contenidos como en planteamiento metodológico.

h) Es muy complicado analizar los diseños de los másteres dada su gran diversidad. La división entre postgrados de carácter profesional e investigador solamente aparece de manera explícita en Francia y de manera implícita en algunos diseños belgas, alemanes y británicos.

i) La presencia de profesionales entre el profesorado de las titulaciones educativas es en general bastante escaso entre las titulaciones universitarias y bastante abundante en las que se desarrollan en la enseñanza superior no universitaria. En algunas titulaciones belgas concerniendo 
a la formación del profesorado, el gobierno lo ha establecido como medida obligatoria.

j) Salvo en Italia y España, en los demás países los criterios de acceso a las titulaciones para los estudiantes no son estrictamente académicos sino que se desprenden de un dossier presentado por el estudiante en el que se incluyen aspectos sociales y preprofesionales (o de voluntariado) de su vida. Esto es mucho más estricto en las titulaciones no universitarias.

k) Los períodos de prácticas existen en casi todas las titulaciones (salvo las francesas y parte de las italianas) aunque su duración y explotación en la titulación es muy diversa, siendo mucho más importantes, como ya se señaló, en las titulaciones socioeducativas que se realizan fuera de la universidad.

La foto final del análisis de las titulaciones educativas en estos seis países europeos sería la heterogeneidad desde una estructura cada vez más homogénea. Esto genera necesariamente algunos cambios especialmente en la mentalidad española, por cuanto alejan la correspondencia entre titulación y mercado laboral. En la mayor parte de los países estudiados, un determinado título no implica un ámbito laboral determinado sino que en la mayoría de las ocasiones genera un abanico de posibilidades de ámbitos de intervención, sin que casi nunca se establezca una relación de exclusividad entre la titulación y los ámbitos. Esta afirmación se matiza más para las profesiones socioeducativas donde la relación estudios-intervención profesional es más estrecha, pero aún en esos mismos no suelen plantearse con exclusividad. Evidentemente lo anteriormente expuesto no se aplica a la formación del profesorado donde los gobiernos establecen las directrices propias a partir de las directrices europeas.

Lo anteriormente expuesto generará algunas dificultades a los colegios profesionales, demasiado habituados a establecer una relación de exclusividad entre una determinada titulación y un ámbito de intervención. Sin duda la llegada de titulados europeos con estudios cuya denominación no se corresponde con la de los estudios españoles les planteará problemas al juzgar la admisión o no de estos titulados, a quienes, en virtud de la propia directiva europea, no podrán impedirles su entrada en el mercado laboral.

Es evidente que la construcción de este espacio europeo de educación superior planteará muchos flecos, aparentemente no muy importantes, pero que crearán no pocas complicaciones en la vida laboral de los nuevos titulados destinados a hacer real esa movilidad personal y profesional por nuestra vieja Europa. 


\section{NOTAS}

1.- Cátedra de Pedagogía en la Facultad de Filosofía y Letras de Madrid (creada en 1901) y Cátedra de Pedagogía Superior en el doctorado de este centro (1904) que fue regida Manuel Bartolomé Cossío hasta 1929 en que desapareció.

2.- En diversas universidades francesas encontraremos DUT (Diplomas universitarios de Tecnología) reconvertidos en DEUST (Diplomas de Estudios Universitarios Científicos y Técnicos) a nivel de BAC +2 , o "Licences Professionnelles" a nivel de BAC+3, en temáticas como animación, educación especializada o educación de adultos.

3.- DEFA: Diploma de Estado relativo a las funciones de la Animación.

4.- BEATEP: Certificado de Estado de Animador y Técnico de la Educación Popular. 


\section{DOCUMENTACIÓN ELECTRÓNICA}

Esteban, L. (2007). Antedecentes históricos de la Facultad de Filosofía y Ciencias de la Educación. Disponible en: http://centros.uv.es/web/centros/C11/data /informacion/presentacion/PDF14.pdf [consulta 2007, 11 de noviembre]

UNIVERSIDADES E INSTITUCIONES FRANCESAS:

Université Paris V La Sorbonne: www.univ-paris5.fr/ [consulta 2007, 28 de noviembre]

Université de Limoges: www.unilim.fr [consulta 2007, 29 de noviembre]

Université de Bordeaux: www.u-bordeaux2.fr [consulta 2007, 2 de diciembre]

Université de Lille: www2.univ-lille2.fr [consulta 2007, 2 de diciembre]

Ecole d'Educateurs Spécialisé de Lille: www.capcampus.com/formation/ filieres/ecole-d-educateurs-specialises [consulta 2007, 2 de diciembre]

Institut Régional de Travail social de París: http://www.irtsparis.asso.fr [consulta 2007, 12 de diciembre]

Le site web de l'éducateur spécialisés: http://www.educateur-specialise.net/ [consulta 2007, 15 de diciembre]

UNIVERSIDADES E INSTITUCIONES DE EDUCACIÓN SUPERIOR BRITÁNICAS:

University of Dundee: www.dundee.ac.uk/ [consulta 2007, 19 de diciembre]

University of Oxford: www.ox.ac.uk [consulta 2007, 19 de diciembre]

University of Kingston: www.kingston.ac.uk [consulta 2007, 19 de diciembre]

University of Southampton: www.soton.ac.uk

[consulta 2007, 20 de diciembre]

Institute of Education University of London: www.ioe.ac.uk [consulta 2007, 20 de diciembre]

UNIVERSIDADES E INSTITUCIONES DE EDUCACIÓN SUPERIOR BELGAS:

Université de Louvain: www.uclouvain.be [consulta 2007, 27 de octubre]

Université libre de Bruxelles: www.ulb.ac.be [consulta 2007, 27 de octubre]
Universiteit Gent: www.ugent.be [consulta 2007, 28 de octubre]

Haute Ecole Léonar de Vinci: www.vinci.be [consulta 2007, 28 de octubre]

Ecole d'Éducateurs de la Province de Liêge: www2.prov-liege.be [consulta 2007, 28 de octubre]

Haute Ecole ISELL: www.isell.be [consulta 2007, 29 de octubre]

UNIVERSIDADES E INSTITUCIONES DE EDUCACIÓN SUPERIOR ITALIANAS:

Universita di Salento: www.unile.it [consulta 2008, 7 de enero]

Università di Bologna: www.unibo.it [consulta 2008, 7 de enero]

Università di Roma III: www.uniroma3.it [consulta 2008, 8 de enero]

Università di Padova: www.unipd.it [consulta 2008, 8 de enero]

Università di Genova: www.unige.it [consulta 2008, 14 de enero]

UNIVERSIDADES E INSTITUCIONES DE EDUCACIÓN SUPERIOR ALEMANAS:

Johannes Gutenberg Universität Mainz: www.uni-mainz.de [consulta 2008, 22 de enero]

Freie Universität Berlin: www.fu-berlin.de [consulta 2008, 26 de enero]

Universität Tubingen: www.uni-tuebingen.de [consulta 2008, 26 de enero]

Universität Hamburg: www.uni-hamburg.de [consulta 2008, 27 de enero]

Fachhoschule Kiel www.fh-kiel.de [consulta 2008, 27 de enero]

Fachhoschule Frankfurt: www.fh-frankfurt. de [consulta 2008, 27 de enero]

OTRAS INSTITUCIONES:

Centro de Documentación Educativa Europea: www.eurydice.org

Ministerio de Educación: www.mec.es

Eduso, el Portal de la educación social: www.eduso.net

Asociación europea de educadores sociales: www.aieji.net 


\section{PALABRAS CLAVE}

Pedagogía, Educación Social, metodología comparitivista.

\section{KEY WORDS}

Pedagogy, Social Education, Comparative Methodology.

\section{PERFIL ACADÉMICO DEL AUTOR}

Joan María Senent es profesor Titular del Departamento de Historia de la Educación y Educación Comparada de la Universidad de Valencia y miembro de la comisión de expertos para la elaboración del Libro Blanco de los grados de Pedagogía y Educación Social.

Dirección del autor: Departament d'Educació Comparada i Història de l'Educació.

Universidad de Valencia

Avda. Blasco Ibáñez, 30

46010 Valencia

E-mail: joan.m.senent@uv.es

Fecha recepción del artículo: 11. marzo. 2008

Fecha aceptación del artículo: 08. mayo. 2008 